\title{
Parallel Variable Distribution Algorithm for Constrained Optimization with Nonmonotone Technique
}

\author{
Congying Han, ${ }^{1,2}$ Tingting Feng, ${ }^{2}$ Guoping $\mathrm{He}^{2}$ and Tiande Guo ${ }^{1}$ \\ ${ }^{1}$ School of Mathematical Sciences, University of the Chinese Academy of Sciences, No. 19(A), Yuquan Road, Shijingshan District, \\ Beijing 100049, China \\ ${ }^{2}$ College of Information Science and Engineering, Shandong University of Science and Technology, Qingdao 266510, China
}

Correspondence should be addressed to Congying Han; hancy@ucas.ac.cn

Received 6 September 2012; Accepted 28 February 2013

Academic Editor: Ching-Jong Liao

Copyright (c 2013 Congying Han et al. This is an open access article distributed under the Creative Commons Attribution License, which permits unrestricted use, distribution, and reproduction in any medium, provided the original work is properly cited.

\begin{abstract}
A modified parallel variable distribution (PVD) algorithm for solving large-scale constrained optimization problems is developed, which modifies quadratic subproblem $Q P_{l}$ at each iteration instead of the $Q P_{l}^{0}$ of the SQP-type PVD algorithm proposed by C. A. Sagastizábal and M. V. Solodov in 2002. The algorithm can circumvent the difficulties associated with the possible inconsistency of $Q P_{l}^{0}$ subproblem of the original SQP method. Moreover, we introduce a nonmonotone technique instead of the penalty function to carry out the line search procedure with more flexibly. Under appropriate conditions, the global convergence of the method is established. In the final part, parallel numerical experiments are implemented on CUDA based on GPU (Graphics Processing unit).
\end{abstract}

\section{Introduction}

In this paper, we consider the following nonlinear programming problem:

$$
\begin{aligned}
& \min \quad f(x), \\
& \text { s.t. } \quad c(x) \leq 0,
\end{aligned}
$$

where $f: R^{n} \rightarrow R$ and $c(x): R^{n} \rightarrow R^{m}$ are all continuously differentiable. Suppose the feasible set $X$ in (1) is described by a system of inequality constraints:

$$
Z=\left\{x \in R^{n} \mid c_{i}(x) \leq 0, I=\{i \mid i=1,2, \ldots, m\}\right\} .
$$

In this paper, we give a new algorithm based on the method in [1], which partitions the problem variables $x \in R^{n}$ into $p$ blocks $x_{1}, \ldots, x_{p}$, such that

$$
x=\left(x_{1}, \ldots, x_{p}\right), \quad x_{l} \in R^{n_{l}}, l=1, \ldots, p, \sum_{l=1}^{p} n_{l}=n .
$$

And assume that $X$ has block-separable structure. Specifically,

$$
c(x)=\left\{c_{1}\left(x_{1}\right), c_{2}\left(x_{2}\right), \ldots, c_{p}\left(x_{p}\right)\right\},
$$

$$
c_{l}\left(x_{l}\right): R^{n_{l}} \longrightarrow R^{m_{l}}, \quad l \in\{1,2, \ldots, p\}, \sum_{l=1}^{p} m_{l}=m .
$$

Parallel variable distribution (PVD) algorithm for solving optimization problems was first proposed by Ferris and Mangasarian [2], in 1994, in which the variables are distributed among $p$ processors. Each processor has the primary responsibility for updating its block of variables while allowing the remaining secondary variables to change in a restricted fashion along some easily computable directions. The distinctive novel feature of this algorithm is the presence of the "forget-me-not" term which allows for a change in "secondary" variables. This makes PVD fundamentally different from the block Jacobi [3] and coordinate descent [4] methods. The forget-me-not approach improves robustness and accelerates convergence of the algorithm and is the key to its success. In 1997, Solodov [5] proposed useful generalizations that consist, for the general unconstrained case, of replacing exact global solution of the subproblems by 
a certain natural sufficient descent condition, and, for the convex case, of inexact subproblem solution in the PVD algorithm. Solodov [6] proposed an algorithm applied the PVD approach to problems with general convex constraints directly use projected gradient residual function as the direction and show that the algorithm converges, provided that certain conditions are imposed on the change of secondary variables. In 2002, Sagastizábal and Solodov [1] proposed two new variants of PVD for the constrained case. Without assuming convexity of constraints, but assuming blockseparable structure, they showed that PVD subproblems can be solved inexactly by solving their quadratic programming approximations. This extends PVD to nonconvex (separable) feasible sets and provided a constructive practical way of solving the parallel subproblems. For inseparable constraints, but assuming convexity, they developed a PVD method based on suitable approximate projected gradient directions. The approximation criterion was based on a certain error bound result, and it was readily implementable. In 2011, Zheng et al. [7] gave a parallel SSLE algorithm, in which the PVD subproblems are solved inexactly by serial sequential linear equations, for solving large-scale constrained optimization with block-separable structure. Without assuming the convexity of constraints, the algorithm is proved to be globally convergent to a KKT point. Han et al. [8] proposed an asynchronous PVT algorithm for solving large-scale linearly constrained convex minimization problems with the idea of [9] in 2009, which based on the idea that a constrained optimization problem is equivalent to a differentiable unconstrained optimization problem by introducing the Fischer Function. And in particular, different from [9] the linear rate of convergence does not depend on the number of processors.

In this paper, we use [1] as our main reference on SQPtype PVD method for problems (1) and (4). Firstly, we introduce the algorithm in [1] simply. The original problem is distributed into $p$ parallel subproblems which treatment among $p$ parallel processors. The algorithm of [1] may result in that the linear constraints in quadratic programming subproblems are inconsistent or the solution of quadratic programming subproblems is unbounded, so that the algorithm may fail. This drawback has been overcome by many researchers, such as [10]. In [1], exact penalty function is used as merit function to carry out the line-search. For the penalty function, as pointed out by Fletcher and Leyffer [11], the biggest drawback is that the penalty parameter estimates could be problematic to obtain. To overcome this drawback, we use a nonmonotone technique to carry out the line search instead of penalty function.

Recent research [12-14] indicates that the monotone line search technique may have some drawbacks. In particular, enforcing monotonicity may considerably reduce the rate of convergence when the iteration is trapped near a narrow curved valley, which can result in very short steps or zigzagging. Therefore, it might be advantageous to allow the iterative sequence to occasionally generate points with nonmonotone objective values. Grippo et al. [12] generalized the Armijo rule and proposed a nonmonotone line search technique for Newton's method which permits increase in function value, while retaining global convergence of the minimization algorithm. In [15], Sun et al. give several nonmonotone line search techniques, such as nonmonotone Armijo rule, nonmonotone Wolfe rule, nonmonotone F-rule, and so on. Several numerical tests show that the nonmonotone line search technique for unconstrained optimization and constrained optimization is efficient and competitive [12$14,16]$. Recently, [17] gives a method to overcome the drawback of zigzagging with nonmonotone line search technique to determine the step length, which makes the algorithm more flexible.

In this paper, we combine [1] with the ideas of [10,17] and propose an infeasible SQP-type Parallel Variable Distribution algorithm for constrained optimization with nonmonotone technique.

The paper is organized as follows. The algorithm is presented in Section 2. In Section 3, under mild assumptions, some global convergence results are proved. The numerical results are shown in Section 4. And conclusions are given in the last section.

\section{A Modified SQP-Type PVD Method with Nonmonotone Technique}

To describe our algorithm, we first give the modified quadratic subproblems of SQP method. Given $x^{k} \in R^{n}$, we distribute it into $p$ blocks, as the iterate $x_{l}^{k} \in R^{n_{l}}, l=1 \ldots, p, H_{l} \in$ $R^{n_{l} \times n_{l}}, l=1,2, \ldots, p$ denote an approximate Hessian.

We use $z_{l} \in R$ to perturb the subproblem of $Q P_{l}^{0}$ of [1] and give a new subproblem $Q P_{l}$ instead of it. Consider

$$
\begin{aligned}
& Q P_{l}\left(x_{l}^{k}, H_{l}^{k}\right) \\
& = \begin{cases}\min _{\left(d_{l}, z_{l}\right) \in R^{n+1}} & z_{l}+\frac{1}{2} d_{l}^{T} H_{l}^{k} d_{l} \\
\text { s.t. } & \left(g_{l}^{k}\right)^{T} d_{l} \leq z_{l}, \\
& c_{i}\left(x_{l}^{k}\right)+\nabla c_{i}\left(x_{l}^{k}\right)^{T} d_{l} \leq z_{l}, \\
& i \in I_{l}=\left\{1, \ldots, m_{l}\right\} .\end{cases}
\end{aligned}
$$

For convenience, given $x$, the $l$ th block of $\nabla f(x)$ will be denoted by

$$
g_{l}=\left(\begin{array}{ll}
I_{n_{l} \times n_{l}} & 0_{\bar{n}_{l} \times \bar{n}_{l}}
\end{array}\right) \nabla f(x) .
$$

In (5), $g_{l}^{k}$ is $g_{l}$ in step $k$. Note that (5) is always feasible for $d_{l}=0, z_{l}=\max _{i \in I_{l}}\left\{c_{i}\left(x_{l}^{k}\right), 0\right\}$. To test whether constraints are satisfied or not, we denote the violation function $h(x)$ as follows:

$$
h(x)=\left\|c(x)^{+}\right\|
$$

where $c_{i}(x)^{+}=\max \left\{c_{i}(x), 0\right\}, i \in I,\|\cdot\|$ denotes the Euclidean norm on $R^{m}$. It is easy to see that $h(x)=0$ if and only if $x$ is a feasible point $(h(x)>0$ if and only if $x$ is infeasible).

We describe our modified PVD algorithm as follows.

Algorithm A. Consider the following. 
Step 0. Start with any $x_{0} \in R^{n}$. Choose parameters $u \in(0$, $1 / 2), \beta \in(1 / 2,1), \gamma \in(0,1)$, positive integer $M>0$, and positive definite matrices $H_{l}^{0} \in R^{n_{l} \times n_{l}}, l \in\{1,2, \ldots, p\}$. Set $k=0, m(k)=0$.

Having $x_{k}$, check a stopping criterion. If it is not satisfied, compute $x_{k+1}$ as follows.

Step 1. Parallelization. For each processor $l \in\{1,2, \ldots, p\}$, solve subproblem (5) to get $\left(d_{l}^{k}, z_{l}^{k}\right)$. If $d_{l}^{k}=0, l=1, \ldots, p$ stop, otherwise return to Step 2.

Step 2. Synchronization. Set

$$
\begin{gathered}
d_{k}=\left(d_{1}^{k}, \ldots, d_{p}^{k}\right), \quad g_{k}^{T}=\left(\left(g_{1}^{k}\right)^{T}, \ldots,\left(g_{p}^{k}\right)^{T}\right), \\
H_{k}=\left(\begin{array}{ccc}
H_{1}^{k} & & \\
& \ddots & \\
& & H_{p}^{k}
\end{array}\right) .
\end{gathered}
$$

If $g_{k}^{T} d_{k} \leq-(1 / 2) d_{k}^{T} H_{k} d_{k}$, return to Step 3; otherwise, set $\lambda_{k}=$ 1 and return to Step 4.

Step 3. Choose $\lambda_{k}$ which is the largest one in the sequence $\left\{1, \gamma, \gamma^{2}, \ldots\right\}$ satisfying:

$$
f\left(x_{k}+\lambda d_{k}\right) \leq \max _{0 \leq j \leq m(k)}\left[f\left(x_{k-j}\right)\right]-\lambda \mu d_{k}^{T} H_{k} d_{k} .
$$

Step 4. Set $x_{k+1}=x_{k}+\lambda_{k} d_{k}$. Let $\bar{h}=\max _{0 \leq j \leq m(k)}\left[h\left(x_{k-j}\right)\right]$. If $h\left(x_{k+1}\right) \leq \beta \bar{h}$, then set $m(k+1)=\min \{m(k)+1, M\}$, update $H_{l}^{k}$ to $H_{l}^{k+1}, l=1, \ldots, p$, set $k=k+1$, and go to Step 1 . Otherwise, let $k=k+1$, call Restoration Algorithm of [17] to obtain $x_{k}^{r}$, let $x_{k}=x_{k}^{r}, m(k)=m(i)$, and go to Step 1 .

Remark 1. In Step 3 of Algorithm A, the nonmonotone parameter $m(k)$ satisfies

$$
\begin{array}{r}
m(0)=0, \quad 0 \leq m(k) \leq \min \{m(k-1)+1, M\}, \\
k \geq 1 .
\end{array}
$$

For the convenience, we denote $f\left(x_{l(k)}\right)=\max _{0 \leq j \leq m(k)}\left[f\left(x_{k-j}\right)\right]$, where $k-m(k) \leq l(k) \leq \mathrm{k}$.

In a restoration algorithm, we aim to decrease the value of $h(x)$ more precisely, we will use a trust region type method to obtain $h\left(x_{k}\right) \rightarrow 0, k \rightarrow \infty$ by the help of the nonmonotone technique. Let

$$
\begin{gathered}
M_{k}^{i}(d)=h\left(x_{k}^{i}\right)-h\left(x_{k}^{i}+d\right), \\
\Psi_{k}^{i}(d)=h\left(x_{k}^{i}\right)-\left\|\left(c\left(x_{k}^{i}\right)+\nabla c\left(x_{k}^{i}\right)^{T} d\right)^{+}\right\|,
\end{gathered}
$$

and $r_{k}^{i}=M_{k}^{i}(d) / \Psi_{k}^{i}(d)$.

Algorithm $\mathrm{B}$ is similar to the restoration phase given by $\mathrm{Su}$ and $\mathrm{Yu}$ [17], we describe the Restoration Algorithm as follows.

Algorithm B. Consider the following.
Step 0. Assume $x_{k}^{0}=x_{k}, \Delta^{0}>0, i=0, \eta \in(0,1), m(i)=0$.

Step 1. If $h\left(x_{k}^{i}\right) \leq \beta \bar{h}$, then $x_{k}^{r}=x_{k}^{i}$ stop.

Step 2. Compute

$$
\begin{array}{cc}
\max & \Psi_{k}^{i}(d) \\
\text { s.t. } & \|d\| \leq \Delta^{i}
\end{array}
$$

to get $d_{k}^{i}$. Calculate $r_{k}^{i}$.

Step 3. If $r_{k}^{i} \leq \eta$, then let $x_{k}^{i+1}=x_{k}^{i}, \Delta^{i+1}=\Delta^{i} / 2, i=i+1$, $m(i)=\min \{m(i-1)+1, M\}$ and go to Step 2 .

Step 4. If $r_{k}^{i}>\eta$, then let $x_{k}^{i+1}=x_{k}^{i}+d_{k}^{i}, \Delta^{i+1}=2 \Delta^{i}, i=i+1$, $m(i)=\min \{m(i-1)+1, M\}$ and go to Step 1 .

\section{The Convergence Properties}

To prove the global convergence of Algorithm A, we make the following assumptions.

\section{Assumptions}

(A1) The iterate $\left\{x_{k}\right\}$ remains in compacted subset $S \subset R^{n}$.

(A2) The objective function $f$ and the constraint functions $c_{j}(j \in I)$ are twice continuously differentiable on $R^{n}$.

(A3) For all $x_{l} \in R^{n_{l}}$, the set $\left\{\nabla c_{i}\left(x_{l}\right): i \in I_{l}\left(x_{l}\right)\right\}$ is linearly independent, where $I_{l}\left(x_{l}\right)=\left\{i \in I_{l}: c_{i}\left(x_{l}\right)=\right.$ $\left.\Phi_{l}\left(x_{l}\right), \Phi_{l}\left(x_{l}\right)=\max _{i \in I_{l}}\left\{c_{i}\left(x_{l}\right), 0\right\}\right\}$.

(A4) There exist two constants $0<a \leq b$ such that $a\left\|d_{l}\right\|^{2} \leq$ $d_{l}^{T} H_{l}^{k} d_{l} \leq b\left\|d_{l}\right\|^{2}, l=1, \ldots, p$ for all iteration $k$ and $d_{l} \in R^{n_{l}}$.

(A5) The solution of problem (12) satisfies

$$
\begin{aligned}
\Psi_{k}^{i}(d) & =h\left(x_{k}^{i}\right)-\left\|\left(c\left(x_{k}^{i}\right)+\nabla c\left(x_{k}^{i}\right)^{T} d\right)^{+}\right\| \\
& \geq \beta_{2} \Delta^{i} \min \left\{h\left(x_{k}^{i}\right), \Delta^{i}\right\},
\end{aligned}
$$

where $\beta_{2}$ is a constant.

Remark 2. Assumptions (A1) and (A2) are the standard assumptions. (A3) is the LICQ constraint qualification. (A4) plays an important role in obtaining the convergence results. (A5) is the sufficient reduction condition which guarantees the global convergence in a trust region method. Under the assumptions, $f$ is bounded below and the gradient function $g_{l}, l=1, \ldots, p$ is uniformly continuous in $S_{l}$, where $S=$ $\left(S_{1}, \ldots, S_{p}\right)$.

Remark 3. We can use quasi-Newton BFGS methods to update $H_{l}^{k}$, different from [18], we can use a small modification of $y_{l}^{k}$ to make $H_{l}^{k}$ reserve positive definite according to the formula (33) of [17]. 
Similar to Lemma 1 in [19], we can get the following conclusions.

Lemma 4. Suppose (A1)-(A4) hold, $H_{l}^{k}, l=1, \ldots, p$ is a symmetric positive definite, then $d_{l}^{k}$ is well defined and $\left(d_{l}^{k}, z_{l}^{k}\right)$ is the unique KKT point of (5). Furthermore, $d_{l}^{k}$ is bounded over compact subsets of $S_{l} \times P_{l}$, where $P_{l}$ is the set of symmetric positive definite $n_{l} \times n_{l}$ matrices.

Proof. First note that the feasible set for (5) is nonempty, since $\left(d_{l}, z_{l}\right)=\left(0, \max _{i \in I_{l}}\left\{c_{i}\left(x_{l}\right), 0\right\}\right)$ is always feasible. It is clear that, if $\left(d_{l}^{k}, z_{l}^{k}\right)$ is a solution to (5) if an only if $d_{l}^{k}$ solves the unconstrained problem

$$
\begin{aligned}
& \min _{d_{l} \in R^{n_{l}}} \frac{1}{2} d_{l}^{T} H_{l}^{k} d_{l} \\
& \quad+\max \left\{\left(g_{l}^{k}\right)^{T} d_{l}, \max _{i \in I_{l}}\left\{c_{i}\left(x_{l}^{k}\right)+\nabla c_{i}\left(x_{l}^{k}\right)^{T} d_{l}\right\}\right\}, \\
& z_{l}=\max \left\{\left(g_{l}^{k}\right)^{T} d_{l}, \max _{i \in I_{l}}\left\{c_{i}\left(x_{l}^{k}\right)+\nabla c_{i}\left(x_{l}^{k}\right)^{T} d_{l}\right\}\right\} .
\end{aligned}
$$

Since the function being minimized in (14) is strictly convex and radially unbounded, it follows that $d_{l}^{k}$ is well defined and unique as a global minimizer for the convex problem (5) and thus unique as a KKT point for that problem. So we have

$$
\begin{gathered}
H_{l}^{k} d_{l}^{k}+v g_{l}^{k}+\nabla c_{l}\left(x_{l}^{k}\right)^{T} \mu_{l}^{k}=0, \\
1-v-\sum_{i=1}^{m_{l}} u_{i}=0,
\end{gathered}
$$

due to (17) and $u_{i} \geq 0, v \geq 0$, we have $u_{i}, i \in I_{l}$ is bounded. Since $f, c_{i}$ is twice continuously differentiable and assumption (A1) holds, for all $x_{l} \in S_{l}, \|-\left(v g_{l}+\nabla c_{l}\left(x_{l}^{k}\right)^{T} \mu_{l}^{k} \|\right.$ is bounded, set the maximum is $M$. For (16), $\left\|H_{l}^{k} d_{l}^{k}\right\| \leq M$ and combining with Assumption (A4), we have $a\left\|d_{l}^{k}\right\|^{2} \leq$ $d_{l}^{k T} H_{l}^{k} d_{l}^{k} \leq\left\|d_{l}^{k}\right\|\left\|H_{l}^{k} d_{l}^{k}\right\| \leq M\left\|d_{l}^{k}\right\|$, so that $\left\|d_{l}^{k}\right\| \leq M / a$; therefore, $d_{l}^{k}$ is bounded on $S_{l} \times P_{l}$.

Lemma 5. Suppose that $x_{l}^{k} \in R^{n_{l}}, l=1, \ldots, p, H_{l}^{k}$ is positive definite matrix, and $\left(d_{l}^{k}, z_{l}^{k}\right)$ is the solution of $Q P_{l}$. Then we have the results (B1) and (B2).

(B1) The following inequality holds:

$$
z_{l}^{k} \leq \Phi_{l}\left(x_{l}^{k}\right)-\frac{1}{2}\left(d_{l}^{k}\right)^{T} H_{l}^{k} d_{l}^{k}, \quad l=1, \ldots, p,
$$

where $\Phi_{l}\left(x_{l}\right)=\max _{i \in I_{l}}\left\{c_{i}\left(x_{l}\right), 0\right\}$.

(B2) If $d_{l}^{k}=0$ then $z_{l}^{k}=0, l=1, \ldots, p$, and $x_{k}=$ $\left(x_{1}^{k}, \ldots, x_{p}^{k}\right)$ is a KKT point of problem (1).

Proof. (B1) Since $\bar{d}_{l}=0, \bar{z}_{l}=\max _{i \in I_{l}}\left\{c_{i}\left(x_{l}\right), 0\right\}$ is a feasible point of $Q P_{1}$, from the optimality of $\left(d_{l}^{k}, z_{l}^{k}\right)$, we have

$$
z_{l}^{k}+\frac{1}{2}\left(d_{l}^{k}\right)^{T} H_{l}^{k} d_{l}^{k} \leq \bar{z}_{l}+0=\max _{i \in I_{l}}\left\{c_{i}\left(x_{l}^{k}\right), 0\right\} .
$$

Together with the definination of $\Phi_{l}\left(x_{l}^{k}\right)$, implies that (18) holds.

(B2) Since $\left(d_{l}^{k}, z_{l}^{k}\right)$ is the solution of (5), there exists $v \in R$ and $\mu \in R^{m_{l}}$ such that the KKT condition of (5), while $d_{l}^{k}=0$, we have

$$
\begin{gathered}
v g_{l}^{k}+\nabla c_{l}\left(x_{l}^{k}\right)^{T} \mu_{l}^{k}=0, \\
1-v-\sum_{i=1}^{m_{l}} u_{i}^{k}=0, \\
-z_{l}^{k} \leq 0, \quad c_{i}\left(x_{l}^{k}\right)-z_{l}^{k} \leq 0, \quad i \in I_{l}, \\
v z_{l}^{k}=0, \quad v \geq 0, \\
u_{i}^{k}\left[c_{i}\left(x_{l}^{k}\right)-z_{l}^{k}\right]=0, \quad u_{i}^{k} \geq 0, \quad i \in I_{l} .
\end{gathered}
$$

By the definition of $\Phi_{l}\left(x_{l}^{k}\right)$ and (22), $\Phi_{l}\left(x_{l}^{k}\right) \leq z_{l}^{k}$. Then, from (18) and $d_{l}^{k}=0, \Phi_{l}\left(x_{l}^{k}\right) \geq z_{l}^{k}$. From (24), it follows that

$$
u_{i}^{k}=0, \quad \forall i \notin I_{l}^{0}=\left\{i \in I_{l}: c_{i}\left(x_{l}^{k}\right)=\Phi_{l}\left(x_{l}^{k}\right)\right\} .
$$

Hence, it follows from Assumption (A3) Together with (20) and (21) that $v>0$. For (23) we have $z_{l}^{k}=0$.

From (22) and (24), we have

$$
\begin{gathered}
u_{i}^{k} c_{i}\left(x_{l}^{k}\right)=0, \quad u_{i}^{k} \geq 0, \quad i \in I_{l}, \\
c_{i}\left(x_{l}^{k}\right) \leq 0, \quad i \in I_{l},
\end{gathered}
$$

due to (20) and set $\rho_{i}^{k}=u_{i}^{k} / v$, we have

$$
g_{l}^{k}+\nabla c_{l}\left(x_{l}^{k}\right)^{T} \rho_{l}^{k}=0 .
$$

Taking into account separability of constraints $c(x)$, and let $I=\cup_{l=1}^{p} I_{l}$, then

$$
\begin{gathered}
\rho_{i}^{k} c_{i}\left(x_{k}\right)=0, \quad \rho_{i}^{k} \geq 0, \quad i \in I, \\
c_{i}\left(x_{k}\right) \leq 0, \quad i \in I, \\
g_{k}+\nabla c_{i}\left(x_{k}\right)^{T} \rho_{k}=0
\end{gathered}
$$

that is, $x_{k}$ is a KKT point of (1).

Lemma 6 (see [17, Lemma 3]). In Step 3, the line search procedure is well defined.

Lemma 7 (see [17, Lemma 4]). Under Assumption (A5), the Restoration Algorithm (Algorithm B) terminates finitely.

From Lemmas 6 and 7, we know that the Algorithm A and Algorithm B are well implemented.

The following Lemma implies that every cluster point of $\left\{x_{k}\right\}$ which generated by Algorithm $\mathrm{A}$ is a feasible point of problem (1).

Lemma 8. Let $\left\{x_{k}\right\}$ be an infinite sequence generated by Algorithm $A$, then $h\left(x_{k}\right) \rightarrow 0(k \rightarrow \infty)$. 
Theorem 9. Suppose $\left\{x_{k}\right\}$ is an infinite sequence generated by Algorithm A, $d_{l}^{k}, l=1, \ldots, p$ is the solution of (5), $d_{k}=$ $\left(d_{1}^{k}, \ldots, d_{p}^{k}\right)$, then $\lim _{k \rightarrow \infty}\left\|d_{l}^{k}\right\|=0, l=1, \ldots, p$.

Proof. By Assumption (A1), there exists a point $x^{*}$ such that $x_{k} \rightarrow x^{*}$ for $k \in K$, where $K$ is an infinite index set. By Algorithm A and Lemma 8, we consider the following two possible cases.

Case I. $K_{0}=\left\{k \in K \mid g_{k}^{T} d_{k} \leq-(1 / 2) d_{k}^{T} H_{k} d_{k}\right\}$ is an infinite index set. In this case, we have

$$
\begin{aligned}
f\left(x_{k}+\lambda_{k} d_{k}\right) & \leq f\left(x_{l(k)}\right)-\lambda_{k} \mu d_{k}^{T} H_{k} d_{k} \\
& =f\left(x_{l(k)}\right)-\lambda_{k} \mu \sum_{l=1}^{p} d_{l}^{k T} H_{l}^{k} d_{l}^{k} .
\end{aligned}
$$

Since $m(k+1) \leq m(k)+1$, we obtain

$$
\begin{aligned}
f\left(x_{l(k+1)}\right) & =\max _{0 \leq j \leq m(k+1)}\left[f\left(x_{k+1-j}\right)\right] \\
& \leq \max _{0 \leq j \leq m(k)+1}\left[f\left(x_{k+1-j}\right)\right] \\
& =\max \left\{f\left(x_{l(k)}\right), f\left(x_{k+1}\right)\right\} \\
& =f\left(x_{l(k)}\right) .
\end{aligned}
$$

Hence for $m(k) \leq M$, it holds

$$
\begin{aligned}
f\left(x_{l(k)}\right) & \leq f\left(x_{l(l(k)-1)}\right)-\lambda_{l(k)-1} \mu d_{l(k)-1}^{T} H_{l(k)-1} d_{l(k)-1} \\
& =f\left(x_{l(l(k)-1)}\right)-\lambda_{l(k)-1} \mu \sum_{l=1}^{p}\left(d_{l}^{l(k)-1}\right)^{T} H_{l}^{l(k)-1} d_{l}^{l(k)-1} .
\end{aligned}
$$

Since $f$ is bounded below, $\left\{f\left(x_{l(k)}\right)\right\}$ converges. Due to (31), we can obtain

$$
\lim _{k \rightarrow \infty} \lambda_{l(k)-1} \mu \sum_{l=1}^{p}\left(d_{l}^{l(k)-1}\right)^{T} H_{l}^{l(k)-1} d_{l}^{l(k)-1}=0 .
$$

By Lemma 6, there exists $\bar{\lambda}>0$ such that $\lambda_{l(k)-1} \geq \bar{\lambda}$. By Assumption (A4), we obtain

$$
\lim _{k \rightarrow \infty}\left\|d_{l}^{l(k)-1}\right\|=0, \quad l=1, \ldots, p .
$$

From the uniform continuity of $f(x)$, this implies that

$$
\lim _{k \rightarrow \infty} f\left(x_{l(k)-1}\right)=\lim _{k \rightarrow \infty} f\left(x_{l(k)}\right) .
$$

Let $\bar{l}(k)=l(k+M+2)$, for any given $j \geq 1$, we can have

$$
\begin{aligned}
& \lim _{k \rightarrow \infty}\left\|d_{l}^{\bar{l}(k)-j}\right\|=0, \quad l=1, \ldots, p, \\
& \lim _{k \rightarrow \infty} f\left(x_{\bar{l}(k)-j}\right)=\lim _{k \rightarrow \infty} f\left(x_{l(k)}\right) .
\end{aligned}
$$

If $j=1$, since $\{\bar{l}(k)\} \subset\{l(k)\}$, (35) and (36) follow from (33) and (34). For a given number $j>1$, we have

$$
\begin{aligned}
& f\left(x_{\bar{l}(k)-j}\right) \\
& \quad \leq f\left(x_{l \bar{l}(k)-j-1)}\right)-\lambda_{\bar{l}(k)-j-1} \mu d_{\bar{l}(k)-j-1}^{T} H_{\bar{l}(k)-j-1} d_{\bar{l}(k)-j-1} .
\end{aligned}
$$

Using the same arguments above, we obtain

$$
\begin{aligned}
& \lim _{k \rightarrow \infty}\left\|d_{l}^{\bar{l}(k)-j-1}\right\|=0, \quad l=1, \ldots, p, \\
& \lim _{k \rightarrow \infty} f\left(x_{\bar{l}(k)-j-1}\right)=\lim _{k \rightarrow \infty} f\left(x_{l(k)}\right) .
\end{aligned}
$$

Therefore (35) and (36) hold for any given $j \geq 1$.

Now for any $k, x_{k+1}=x_{\bar{l}(k)}-\sum_{j=1}^{\bar{l}(k)-k-1} \lambda_{\bar{l}(k)-j} d_{\bar{l}(k)-j}$, from Remark 1, we obtain $\bar{l}(k)=l(k+M+2)<k+M+2$, that is $\bar{l}(k)-k-1 \leq M+1$. Since $d_{\bar{l}(k)-j}=\left\{d_{1}^{\bar{l}(k)-j}, \ldots, d_{p}^{\bar{l}(k)-j}\right\}$ together with (35), we can obtain $\lim _{k \rightarrow \infty}\left\|d_{\bar{l}(k)-j}\right\|=0,1 \leq$ $j \leq \bar{l}(k)-k-1$. Therefore,

$$
\lim _{k \rightarrow \infty}\left\|x_{k+1}-x_{\bar{l}(k)}\right\|=0 .
$$

Since $\left\{f\left(x_{l(k)}\right)\right\}$ admits a limit, by the uniform continuity of $f$ on $S$, it holds

$$
\lim _{k \rightarrow \infty} f\left(x_{k}\right)=\lim _{k \rightarrow \infty} f\left(x_{\bar{l}(k)-1}\right)=\lim _{k \rightarrow \infty} f\left(x_{l(k)}\right) .
$$

Then by the relation (29), we have

$$
\lim _{k \rightarrow \infty} \sum_{l=1}^{p} \lambda_{k} \mu d_{l}^{k T} H_{l}^{k} d_{l}^{k}=0 .
$$

Using the same arguments for deriving (33), we obtain that

$$
\lim _{k \rightarrow \infty}\left\|d_{l}^{k}\right\|=0, \quad l=1, \ldots, p .
$$

Case II. $K_{0}$ is a finite index set, which implies $K_{1}=\{k \in K \mid$ $\left.g_{k}^{T} d_{k}>-(1 / 2) d_{k}^{T} H_{k} d_{k}\right\}$ is an infinite index set.

If $\lim _{k \rightarrow \infty}\left\|d_{l}^{k}\right\|=0, l=1, \ldots, p$ does not hold, then there exists a positive number $\varepsilon>0$ and an infinite index set $K_{2}$, such that $\left\|d_{l}^{k}\right\|>\varepsilon$, for all $k \in K_{2} \subset K_{1}$. Since $\left(d_{l}^{k}, z_{l}^{k}\right)$ is the solution of $Q P_{l}$. By the KKT condition of (5), we have

$$
\begin{gathered}
H_{l}^{k} d_{l}^{k}+v g_{l}^{k}+\nabla c_{l}\left(x_{l}^{k}\right)^{T} \mu_{l}^{k}=0, \\
1-v-\sum_{i=1}^{m_{l}} u_{i}=0, \\
g_{l}^{k T} d_{l}^{k}-z_{l}^{k} \leq 0,
\end{gathered}
$$

$$
u_{i}\left[c_{i}\left(x_{l}^{k}\right)+\nabla c_{i}\left(x_{l}^{k}\right)^{T} d_{l}^{k}-z_{l}^{k}\right]=0, \quad u_{i} \geq 0, i \in \mathrm{I}_{l} .
$$

Since $v \geq 0, u_{i} \geq 0$ and (44), we obtain $0 \leq u_{i} \leq 1$, therefore there exists $\bar{M}_{l}$ satisfied $\left\|u_{l}^{k}\right\| \leq \bar{M}_{l}$. 
By Lemma $8, h\left(x_{k}\right) \rightarrow 0$ implies $h_{l}\left(x_{l}^{k}\right) \rightarrow 0$. Hence there exists $k_{0}$, such that for $k>k_{0}, k \in K_{2}$, it holds

$$
h_{l}\left(x_{l}^{k}\right) \leq \frac{a \varepsilon^{2}}{2 \bar{M}_{l}} \leq \frac{a\left\|d_{l}^{k}\right\|^{2}}{2 \bar{M}_{l}} \leq \frac{d_{l}^{k T} H_{l}^{k} d_{l}^{k}}{2 \bar{M}_{l}}, \quad l=1, \ldots, p .
$$

Consequently, from (43), we deduce

$$
\begin{aligned}
v g_{l}^{k T} d_{l}^{k} & =-d_{l}^{k T} H_{l}^{k} d_{l}^{k}-\mu_{l}^{k T} \nabla c_{l}\left(x_{l}^{k}\right) d_{l}^{k} \\
& =-d_{l}^{k T} H_{l}^{k} d_{l}^{k}+\sum_{i=1}^{m_{l}} u_{i}\left(c_{i}\left(x_{l}^{k}\right)-z_{l}^{k}\right) \\
& \leq-d_{l}^{k T} H_{l}^{k} d_{l}^{k}+\bar{M}_{l} h_{l}\left(x_{l}^{k}\right)-\sum_{i=1}^{m_{l}} u_{i} z_{l}^{k} \\
& \leq-d_{l}^{k T} H_{l}^{k} d_{l}^{k}+\bar{M}_{l} h_{l}\left(x_{l}^{k}\right)-\sum_{i=1}^{m_{l}} u_{i} g_{l}^{k T} d_{l}^{k} .
\end{aligned}
$$

Together with (44), (47), and transpose

$$
\begin{aligned}
g_{l}^{k T} d_{l}^{k} & =\left(\sum_{i=1}^{m_{l}} u_{i}+v\right) g_{l}^{k T} d_{l}^{k} \\
& \leq-d_{l}^{k T} H_{l}^{k} d_{l}^{k}+\bar{M}_{l} h_{l}\left(x_{l}^{k}\right) \\
& \leq-d_{l}^{k T} H_{l}^{k} d_{l}^{k}+\frac{1}{2} d_{l}^{k T} H_{l}^{k} d_{l}^{k} \\
& =-\frac{1}{2} d_{l}^{k T} H_{l}^{k} d_{l}^{k} .
\end{aligned}
$$

Taking into account separability of constraints $c(x)$, then

$$
\begin{aligned}
g_{k}^{T} d_{k} & =\sum_{l=1}^{p} g_{l}^{k T} d_{l}^{k} \\
& \leq-\sum_{l=1}^{p} \frac{1}{2} d_{l}^{k T} H_{l}^{k} d_{l}^{k} \\
& =-\frac{1}{2} d_{k}^{T} H_{k} d_{k},
\end{aligned}
$$

which contradicts the definition of $K_{1}$. The proof is complete.

Theorem 10. Suppose $\left\{x_{k}\right\}$ is an infinite sequence generated by Algorithm $A$ and the assumptions in Theorem 9 hold. Then every cluster point of $\left\{x_{k}\right\}$ is a KKT point of problem (1).

Proof. By Assumption (A2), there exists $x^{*}$, such that $x_{k} \rightarrow$ $x^{*}, k \in K$. From Lemma 8, we have $h\left(x_{k}\right) \rightarrow 0, k \in K$, which means that $x^{*}$ is a feasible point. From Theorem 9 , we have $\lim _{k \rightarrow \infty}\left\|d_{l}^{k}\right\|=0, l=1, \ldots, p$. By Lemma $5, d_{l}^{k} \rightarrow$ 0 implies that $z_{l}^{k} \rightarrow 0, l=1, \ldots, p$, that is, $d_{l}^{*}=0, l=$ $1, \ldots, p, z_{l}^{*}=0, l=1, \ldots, p$.

Compare the KKT condition of (5) with the KKT condition of problem (1), and let $\rho^{*}=\left(\rho_{1}^{*}, \ldots \rho_{p}^{*}\right), \rho_{l}^{*}=\left(u_{i}^{*} / v, i \in\right.$ $\left.I_{l}\right), I=\cup_{l=1}^{p} I_{l}$.
TABLE 1: Results for Algorithm A.

\begin{tabular}{lcccc}
\hline $\begin{array}{l}\text { Problem } 1 \\
\begin{array}{l}n, v \\
\nu=3\end{array}\end{array}$ & $P$ & $\begin{array}{c}\text { Iteration } \\
\text { number }\end{array}$ & $\begin{array}{c}\text { Algorithm A } \\
\text { Iteration number } \\
\text { of } Q P_{l} \text { subproblem }\end{array}$ & $\begin{array}{c}\text { Running } \\
\text { times }\end{array}$ \\
\hline$n=6$ & 2 & 5 & 52 & 0.5 \\
$n=12$ & 4 & 10 & 66 & 0.5 \\
$n=24$ & 8 & 15 & 78 & 1 \\
$n=48$ & 16 & 9 & 46 & 2 \\
$n=96$ & 32 & 6 & 50 & 9 \\
$n=192$ & 64 & 7 & 24 & 13 \\
\hline
\end{tabular}

Taking into account separability of constraints $c(x)$ and $x$, we conclude

$$
\begin{gathered}
g^{*}+\rho^{*} \nabla c^{*}=0, \quad c\left(x^{*}\right) \leq 0, \quad \rho_{i}^{*} \geq 0, \\
\rho_{i}^{* T} c_{i}\left(x^{*}\right)=0, \quad i \in I .
\end{gathered}
$$

Therefore $x^{*}$ is a KKT point of problem (1).

\section{Numerical Results}

To get some insight into computational properties of our approach in Section 2, we considered the same test problems taken from [1]. Choose Problem 1 of [1] as follows:

$$
\begin{array}{ll}
\min & \sum_{i=1}^{p-1} \sum_{j=i+1}^{p} \sum_{t=1}^{v} x_{(i-1) v+t} x_{(j-1) v+t} \\
\text { s.t. } & \sum_{t=1}^{v} x_{(i-1) v+t}^{2}-1=0, \quad i=1, \ldots, p .
\end{array}
$$

In our test, we only verify our convergence theory without comparing with serial algorithms. In the future, we will propose the convergent rate of the parallel algorithm.

Not having access to a parallel computer, we have carried out on Graphics Processing Unit (GPU) to solve them and implement the algorithm on Compute Unified Device Architecture (CUDA) [20]. However, the input and output are implemented by CPU. And all the subproblems are solved on blocks which are constructed into many threads. If there are $p$ blocks in GPU, which is equivalent to $p$ processors in a parallel computer. Many threads of the block can achieve a large number of vector calculations in current block.

We have implemented Algorithm A in C language. All codes were run under Visual Studio 2008 and Cuda 4.0 on the DELL Precision T7400. In Table 1, we report the number of iterations and the running times for Algorithms A on Problem (52). The results in Table 1 confirm that the proposed approach certainly makes sense. We solve the subproblem (5) which is a positive semidefinite quadratic programs by the modified interior-point methods. And the Restoration Algorithm of [17] is solved by Trust-region Approach combined with local pattern search methods. All algorithms are coded by $\mathrm{C}$ language without using any function from optimization Toolbox; hence the results are not the best. 
In Table $1, n$ denotes the dimensions of test problem and $v$ denotes the variable number of one block. There is less iteration number of $\mathrm{QP}_{l}$ subproblem with more numbers of blocks for high-dimensional problem, which shows the algorithm is reliable.

\section{Conclusion}

The PVD algorithm which was proposed in 1994 is used to solve unconstrained optimization problems or has a special case of convex block-separable constraints. In 1997, M. V. Solodov proposed useful generalizations that consist, for the general unconstrained case, of replacing exact global solution of the subproblems by a certain natural sufficient descent condition, and, for the convex case, of inexact subproblem solution in the PVD algorithm. M. V. Solodov proposed a PVD approach in 1998 which applied to problems with general convex constraints directly and show that the algorithm converges. In 2002, C. A. Sagastizábal et al. propose two variants of PVD for the constrained case: without assuming convexity of constraints, but assuming block-separable structure and for inseparable constraints, but assuming convexity. In this paper, we propose the modified algorithm of [1] used to the general constraints but with block-separable structure.

In a word, the algorithm above is a special structure of the objective function or constraints with a special structure. In the further, we will study the parallel algorithm with general inseparable constraints.

\section{Acknowledgments}

This paper was supported by National Natural Science Foundation of China (11101420, 71271204) and other foundations (2010BSE06047). The authors would like to thank Dr. Jiang Zhipeng for valuable work on numerical experiments.

\section{References}

[1] C. A. Sagastizábal and M. V. Solodov, "Parallel variable distribution for constrained optimization," Computational Optimization and Applications, vol. 22, no. 1, pp. 111-131, 2002.

[2] M. C. Ferris and O. L. Mangasarian, "Parallel constraint distribution," SIAM Journal on Optimization, vol. 1, no. 1, pp. 487-500, 1994.

[3] D. P. Bertsekas and J. N. Tsitsiklis, Parallel and Distributed Computation, Prentice Hall, Englewood Cliffs, NJ, USA, 1989.

[4] P. Tseng, "Dual coordinate ascent methods for non-strictly convex minimization," Mathematical Programming, vol. 59, no. 2, pp. 231-247, 1993.

[5] M. V. Solodov, "New inexact parallel variable distribution algorithms," Computational Optimization and Applications, vol. 7, no. 2, pp. 165-182, 1997.

[6] M. V. Solodov, "On the convergence of constrained parallel variable distribution algorithms," SIAM Journal on Optimization, vol. 8, no. 1, pp. 187-196, 1998.

[7] F. Zheng, C. Han, and Y. Wang, "Parallel SSLE algorithm for large scale constrained optimization," Applied Mathematics and Computation, vol. 217, no. 12, pp. 5377-5384, 2011.

[8] C. Han, Y. Wang, and G. He, "On the convergence of asynchronous parallel algorithm for large-scale linearly constrained minimization problem," Applied Mathematics and Computation, vol. 211, no. 2, pp. 434-441, 2009.

[9] M. Fukushima, "Parallel variable transformation in unconstrained optimization," SIAM Journal on Optimization, vol. 8, no. 3, pp. 658-672, 1998.

[10] J. Mo, K. Zhang, and Z. Wei, "A variant of SQP method for inequality constrained optimization and its global convergence," Journal of Computational and Applied Mathematics, vol. 197, no. 1, pp. 270-281, 2006.

[11] R. Fletcher and S. Leyffer, "Nonlinear programming without a penalty function," Mathematical Programming, vol. 91, pp. 239269, 2002.

[12] L. Grippo, F. Lampariello, and S. Lucidi, "A nonmonotone line search technique for Newton's method," SIAM Journal on Numerical Analysis, vol. 23, no. 4, pp. 707-716, 1986.

[13] L. Grippo, F. Lampariello, and S. Lucidi, "A truncated Newton method with nonmonotone line search for unconstrained optimization," Journal of Optimization Theory and Applications, vol. 60, no. 3, pp. 401-419, 1989.

[14] G. Liu, J. Han, and D. Sun, "Global convergence of the BFGS algorithm with nonmonotone linesearch," Optimization, vol. 34, no. 2, pp. 147-159, 1995.

[15] W. Sun, J. Han, and J. Sun, "Global convergence of nonmonotone descent methods for unconstrained optimization problems," Journal of Computational and Applied Mathematics, vol. 146, no. 1, pp. 89-98, 2002.

[16] P. L. Toint, "An assessment of nonmonotone linesearch techniques for unconstrained optimization," SIAM Journal on Scientific Computing, vol. 17, no. 3, pp. 725-739, 1996.

[17] K. Su and Z. Yu, "A modified SQP method with nonmonotone technique and its global convergence," Computers \& Mathematics with Applications, vol. 57, no. 2, pp. 240-247, 2009.

[18] Y.-X. Yuan and W. Sun, Optimization Theory and Methods, Springer, New York, NY, USA, 2006.

[19] C. T. Lawrence and A. L. Tits, "A computationally efficient feasible sequential quadratic programming algorithm," SIAM Journal on Optimization, vol. 11, no. 4, pp. 1092-1118, 2001.

[20] I. Buck, Parallel Programming with CUDA, 2008, http://gpgpu .org/sc2008. 


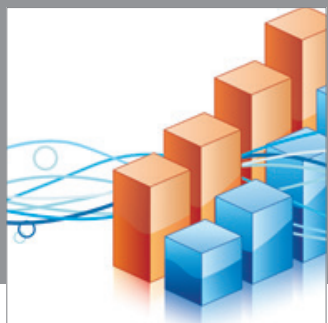

Advances in

Operations Research

mansans

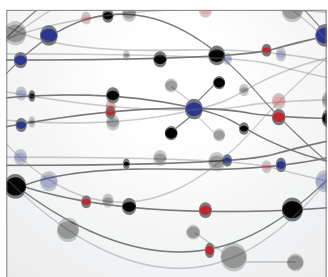

The Scientific World Journal
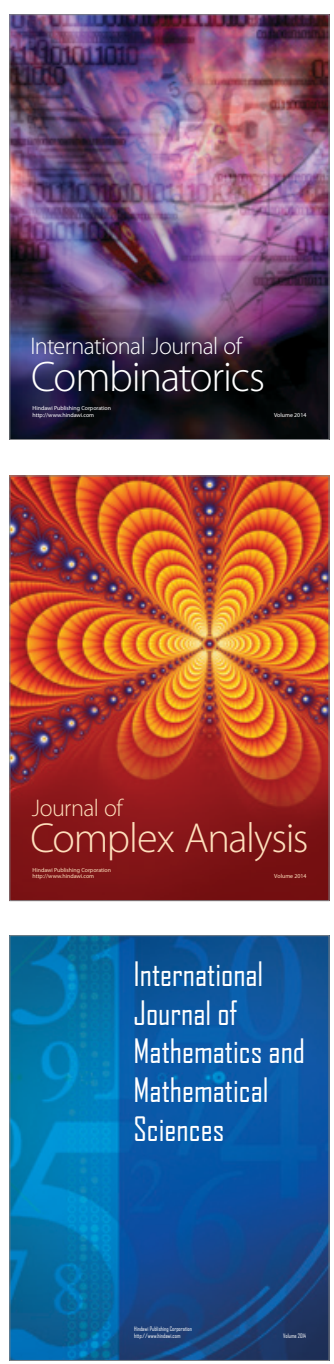
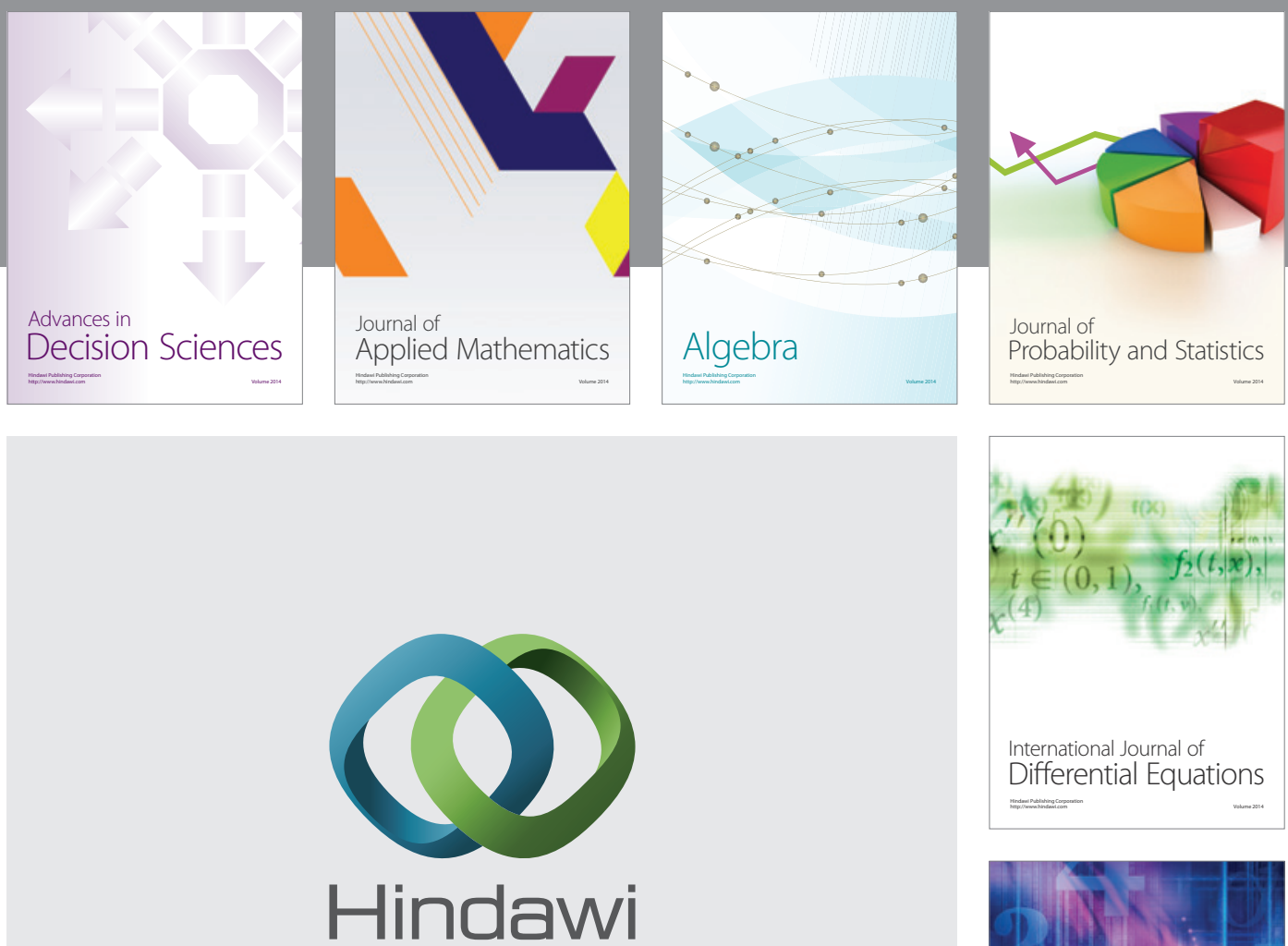

Submit your manuscripts at http://www.hindawi.com
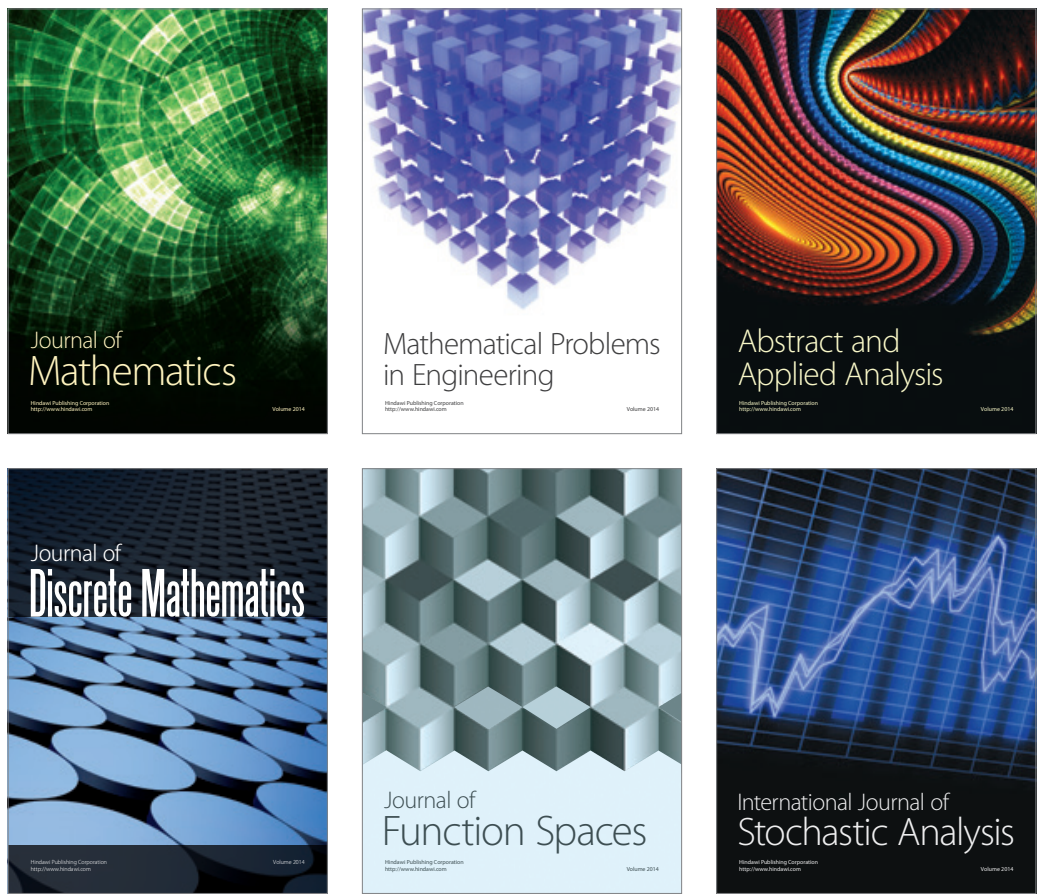

Journal of

Function Spaces

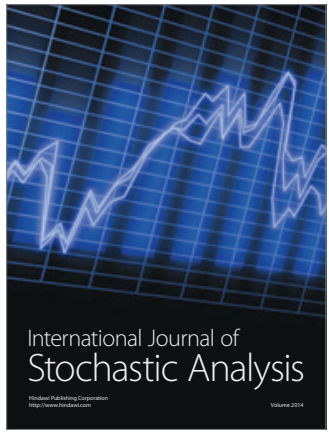

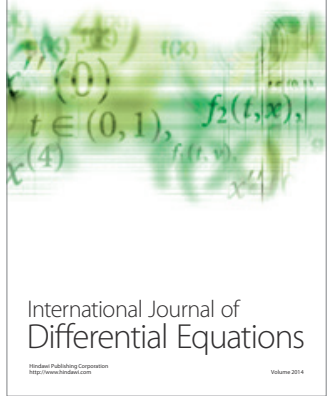
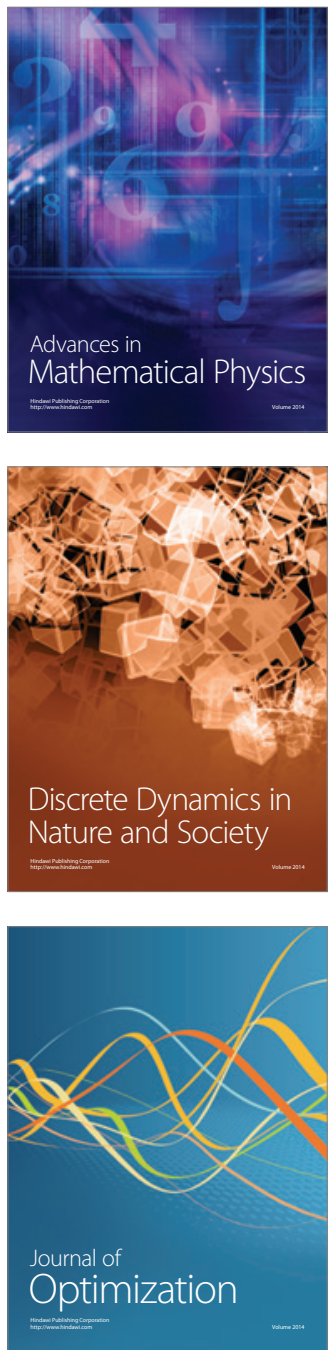